\section{Renal artery stenosis and abdominal aorta aneurysm in patients with pseudoexfoliation syndrome}

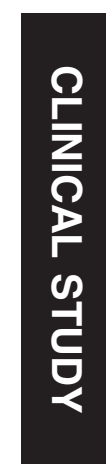

Keywords: pseudoexfoliation syndrome; renal artery stenosis; abdominal aorta aneurysm; Doppler ultrasonography; computed tomographic angiography

\section{Introduction}

Pseudoexfoliation syndrome (PEX) is a systemic disorder that is characterized by the deposition of a fibrillar material in many different tissues over the body together with the ocular tissues. ${ }^{1}$ Its prevalence increases with age. PEX is seen in $30 \%$ of the population over 60 years of age. ${ }^{2}$ The pseudoexfoliative material accumulating in the anterior segment tissues of the eye and blocking aqueous humor drainage is an important cause of open-angle glaucoma. ${ }^{3}$ PEX is a systemic elastosis and it affects the vascular structures. ${ }^{4}$ It is thought to have a role in many systemic vascular disorders such as systemic hypertension, angina pectoris, myocardial infarction, abdominal aorta aneurysm, transient ischemic attack and cerebrovascular events, peripheral vascular diseases, Alzheimer disease, and sensory-neural hearing loss. ${ }^{4-11}$ In PEX, ocular complications are well documented but the clinical importance of systemic manifestations is unknown.

Systemic hypertension is an important cause of morbidity and mortality that affects almost $10 \%$ of the population. ${ }^{12,13}$ Renovascular problems are responsible for $3-5 \%$ of all systemic hypertension. ${ }^{12-14} \mathrm{~A}$ decrease in the diameter of the renal artery $>50-60 \%$ is hemodynamically important and may lead to a hypertension that cannot be easily controlled by medical therapies. This may end up with renal insufficiency. ${ }^{15}$ In this study, aorta-renal vasculature in patients with and without PEX
Department of Radiology, School of Medicine, Namik Kemal University, Tekirdag, Turkey

${ }^{2}$ Department of Ophthalmology, School of Medicine, Namik Kemal University, Tekirdag, Turkey

${ }^{3}$ Department of Radiology, School of Medicine, Baskent University, Istanbul, Turkey

Correspondence: KA Gonen, Department of Radiology, School of Medicine, Namik Kemal University, Degirmenalti Mah. Gundogan Sitesi B Blok, Daire 6, Tekirdag 59100, Turkey

Tel: + 90533 5109097;

Fax: + 90282 2624732;

E-mail: aysunbalc@yahoo. com

Received: 22 June 2012 Accepted in revised form: 13 February 2013 Published online: 12 April 2013 
was examined with Doppler ultrasonography (US) and computed tomographic (CT) angiography, and the frequency of renal artery stenosis (RAS) and abdominal aorta aneurysm was evaluated.

\section{Materials and methods}

This prospective case-control study included 91 subjects with and without PEX. All participants were 50 years of age or older and they had an intraocular pressure $<21 \mathrm{~mm} \mathrm{Hg}$, a normal optic nerve appearance, and visual field. Exclusion criteria were diabetes mellitus, chronic renal failure, cardiovascular disease (except well-controlled hypertension), a history of smoking, and/or alcohol consumption. The study protocol was approved by the local Ethics Committee of the University of Namik Kemal and performed according to the Helsinki declaration. Written informed consent was obtained from all patients and controls. We certify that all applicable institutional and governmental regulations concerning the ethical use of human volunteers were followed during this research.

Both groups included subjects presenting for routine eye examination with refraction-related symptoms. All participants underwent ophthalmologic examination, including visual acuity (Snellen chart), intraocular pressure measurement by applanation tonometer, slit-lamp biomicroscopy, fundoscopy, and visual field testing (Humprey visual field testing). After pupillary dilation with tropicamide $1 \%$, the observation of typical exfoliative material on the anterior lens capsule and pupillary margin was defined as PEX. Initially, PEX group was constituted by 49 consecutive patients who have PEX in one or both eyes and meet the criteria. Then, control group was constituted by 42 subjects without PEX matched in age with the PEX group (Table 1). Serum creatinine levels were measured in all subjects' blood samples for the evaluation of their renal functions.

After the ophthalmologic examination, all subjects underwent a renal and abdominal aorta Doppler US assessment in the Radiology department. US examination was performed by a specialist in radiology who was experienced in Doppler US and was unaware of the presence or absence of PEX in the participants (KAG). Following 6-12 h fasting, Doppler US was carried out by using the Acuson X300 ultrasound imaging system (Siemens, Mountain View, CA, USA). The originating levels of the main renal arteries from the abdominal aorta (orifice) and their proximal segments and the renal entry points (hilar) were assessed. The maximum diameter and peak systolic velocity (PSV) value of the aorta were measured. The abdominal aorta and the kidneys were carefully evaluated both in transverse and sagittal planes for visualization of possible duplicated renal artery.
Table 1 The demographic and laboratory data of the subjects

\begin{tabular}{lccc}
\hline Parameters & $\begin{array}{c}\text { PEX group } \\
(\mathrm{n}=49)\end{array}$ & $\begin{array}{c}\text { Control group } \\
(\mathrm{n}=42)\end{array}$ & P-value \\
\hline Age, year & & & 0.443 \\
Mean \pm SD (range) & $71.1 \pm 10.3(51-90)$ & $69.5 \pm 9.0(50-87)$ & \\
Distribution, $n(\%)$ & & & \\
$50-59$ & $6(12)$ & $5(12)$ & \\
$60-69$ & $15(31)$ & $13(31)$ & \\
$70-79$ & $20(41)$ & $17(41)$ & \\
$80+$ & $8(16)$ & $7(16)$ & \\
& & & \\
Sex, M/F & $27 / 22$ & $20 / 22$ & 0.413 \\
Serum creatinine (mg/d) & $0.81 \pm 0.28$ & $0.79 \pm 0.22$ & 0.690 \\
Hypertension, $n(\%)$ & $17 / 49(34.7)$ & $16 / 42(38.1)$ & 0.671 \\
\hline
\end{tabular}

Abbreviations: F, female; M, male; $n$, patients; PEX, pseudoexfoliation.

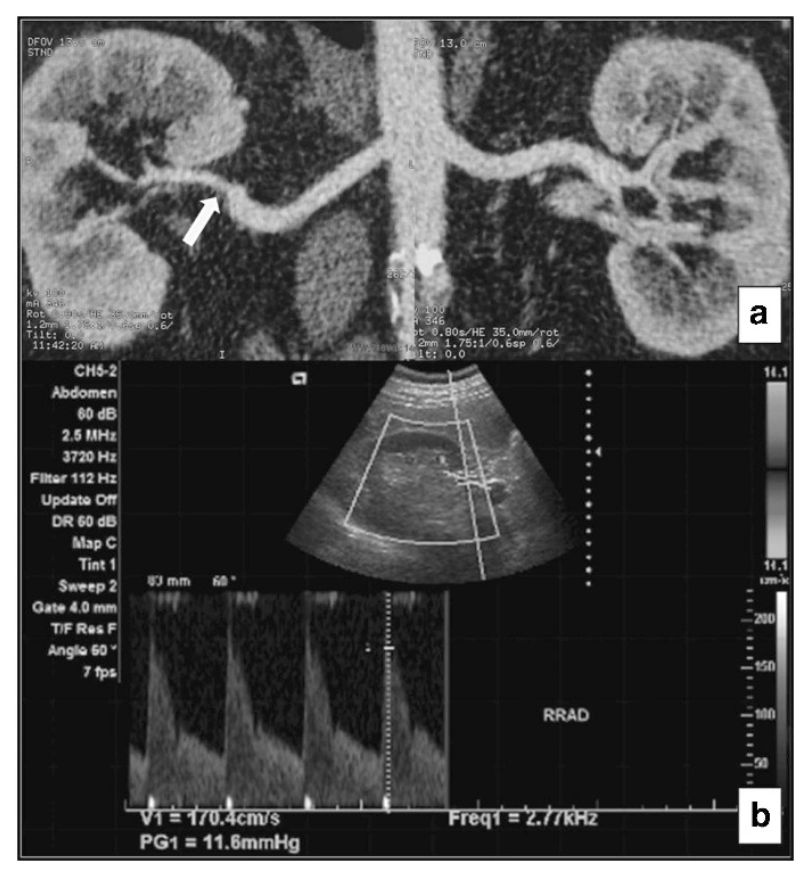

Figure 1 A 64-year-old male patient with significantly stenotic right renal artery in PEX group. (a) CT angiography demonstrated a stenosis (arrow) of the distal right renal artery. The left renal artery was normal. (b) Direct sonographic examination of distal right renal artery shows PSV of $170 \mathrm{~cm} / \mathrm{s}$, indicating significant stenosis.

Spectral Doppler analysis was used for both renal arteries for obtaining the PSV values primarily from the orifice and the hilar levels and the proximal and mid portions that could have been visualized. High-velocity flow points that were thought to be due to stenosis were recorded. The renal artery PSV (RPSV)/abdominal aorta PSV (APSV) ratio (RAR) was calculated. Patients who had RPSV value over $150 \mathrm{~cm} / \mathrm{s}$ and/or RAR value $>3$, and abdominal aorta diameter $>3 \mathrm{~cm}$, were performed for a renal and abdominal aorta CT angiography examination (Figure 1). ${ }^{14,16,17}$ The CT angiography was carried out using a 16-slice multislice CT device (Bright 
Speed 16; General Electric Medical Systems Co., Ltd., Milwaukee, WI, USA) following at least $6 \mathrm{~h}$ of fasting. The patients were injected non-ionic contrast media (Iomeprol, Bracco, Italy) intravenously, and axial images with thickness of $0.625 \mathrm{~mm}$ were obtained. The angiographic images were obtained in another work station by using the AutoBone XPress Program (General Electric Medical Systems Co., Ltd.). The RAS regions were established and a degree of stenosis was calculated. The diameter of the widest part of the abdominal aorta was recorded. The images were evaluated by another radiology specialist (BG) who was blinded to the presence or absence of PEX in the patients.

Statistical analysis was performed with SPSS for Windows 16.0 (Statistical Product and Service Solutions, Inc., Chicago, IL, USA) package program. In descriptive analysis, the measurement variables are given as average and SD. The differences between groups were assessed by using $\chi^{2}$-test and independent sample $t$-tests. $P$-values of $<0.05$ were considered statistically significant.

\section{Results}

The demographic and laboratory data were summarized in Table 1. There were no statistically significant differences in age, gender, serum creatinine levels, and hypertension between patients with PEX and control subjects $(P>0.05)$.

In the orifices and hilar segments of renal artery, no significant difference was seen in the mean PSV between PEX and control groups $(P>0.05)$. However, the mean APSV was significantly different between the two groups $(P<0.046)$ (Table 2$)$. The RAR values were comparable between the groups $(P>0.05)$. When the patients with and without hypertension were evaluated separately, no significant differences were obtained within the groups or among the two groups in terms of RPSV of hilar, RPSV of orifice, APSV, and RAR $(P>0.05)$ (Table 2$)$.

To confirm the RAS and/or abdominal aorta aneurysm, CT angiography was performed in 16 patients who had met the criteria above. Thirteen out of these 16 patients were in the PEX group and 3 were in the control group. RAS (50\% or more) was observed in 9 (18.4\%) patients with PEX and in only 1 (2.4\%) control subject $(P=0.017)$. Six out of the nine patients with PEX and one control subject with RAS had hypertension. When the hypertensive patients were considered as a single group, the RAS was observed in 6 out of 17 (35.3\%) subjects with PEX and in 1 out of 16 (6.3\%) control subjects $(P=0.041)$. On the other hand, abdominal aorta aneurysm $(>3 \mathrm{~cm})$ was observed in 4 of these $49(8.2 \%)$ patients with PEX and in none of these $42(0 \%)$ control subjects $(P=0.061)$. Two out of the four patients with abdominal aorta aneurysm had hypertension (Table 3). One patient with an abdominal aorta aneurysm of $6 \mathrm{~cm}$ in diameter who had PEX but was not hypertensive has undergone a surgical intervention (Figure 2).

\section{Discussion}

In this study, renal arteries and abdominal aorta of the subjects with or without PEX have been evaluated by Doppler US and CT angiography. When compared with the control group, the number of patients with RAS in PEX group was found to be significantly higher.

PEX is a clinical situation that gives rise to both ocular and systemic morbidity. Postmortem investigations have put forward the deposition of pseudoexfoliation material in many tissues and organs other than the eyes. $1,7,18,19$ The pseudoexfoliation material that is basically a glycoprotein-proteoglycan complex is histologically similar to the basement membrane. It has been suggested

Table 2 Doppler ultrasound parameters of the subjects

\begin{tabular}{lcccr}
\hline Groups & RPSV of orifice $(\mathrm{cm} / \mathrm{s})$ & RPSV of hilar $(\mathrm{cm} / \mathrm{s})$ & APSV $(\mathrm{cm} / \mathrm{s})$ & RAR \\
\hline PEX & & & & \\
$\quad$ Total $^{\mathrm{a}}, n=98$ & $88.3 \pm 30.3(42-251)$ & $91.7 \pm 26.1(50-175)$ & $76.0 \pm 30.7(30-208)$ & $1.26 \pm 0.56(0.33-3.18)$ \\
HT $(+)^{\mathrm{b}}, n=34$ & $87.0 \pm 29.7(52-172)$ & $96.9 \pm 26.5(53-169)$ & $76.9 \pm 38.8(30-208)$ & $1.23 \pm 0.65(0.33-3.02)$ \\
HT $(-)^{\mathrm{c}}, n=64$ & $89.0 \pm 31.0(42-251)$ & $89.4 \pm 25.8(50-175)$ & $75.5 \pm 25.7(43-153)$ & $1.28 \pm 0.52(0.42-3.18)$ \\
& & & & \\
Control & & & \\
$\quad$ Total $^{\mathrm{d}}, n=84$ & $79.5 \pm 21.1(44-138)$ & $93.0 \pm 22.6(48-142)$ & $65.2 \pm 17.5(40-135)$ & $1.23 \pm 0.38(0.61-2.17)$ \\
HT $(+)^{\mathrm{e}}, n=32$ & $76.5 \pm 22.8(44-138)$ & $87.9 \pm 20.9(56-128)$ & $67.0 \pm 16.3(40-99)$ & $1.14 \pm 0.39(0.66-1.93)$ \\
HT $(-)^{\mathrm{f}}, n=52$ & $81.4 \pm 20.0(50-120)$ & $96.5 \pm 23.2(48-142)$ & $64.0 \pm 18.4(41-135)$ & $1.29 \pm 0.38(0.61-2.17)$ \\
\hline
\end{tabular}

Abbreviations: APSV, abdominal aorta peak systolic velocity; HT, hypertension; $n$, renal artery; PEX, pseudoexfoliation; RAR, renal-to-aortic ratio; RPSV, renal artery peak systolic velocity.

Independent samples $t$-test, RPSV of orifice, a $v s \mathrm{~d}, P=0.314 ; \mathrm{b}$ vs $\mathrm{c}, P=0.586$; e $v s \mathrm{f}, P=0.778$; $\mathrm{b}$ vs e, $P=0.338$; $\mathrm{c}$ vs $\mathrm{f}, P=0.653$.

RPSV of hilar, a vs $\mathrm{d}, P=0.794 ; \mathrm{b}$ vs c, $P=0.511$; e vs f, $P=0.343$; $\mathrm{b}$ vs e, $P=0.309$; $\mathrm{c}$ vs $\mathrm{f}, P=0.640$.

APSV, a vs d, $P=0.046$; b vs c, $P=0.881$; e vs $\mathrm{f}, P=0.601$; b vs e, $P=0.370$; c vs $\mathrm{f}, P=0.065$.

RAR, a vs d, $P=0.757$; b vs c, $P=0.634$; e vs $\mathrm{f}, P=0.376$; b vs e, $P=0.662$; c vs $\mathrm{f}, P=0.366$. 
Table 3 CT angiography findings in patients with RAS and/or abdominal aorta aneurysm

\begin{tabular}{|c|c|c|c|c|c|c|c|c|c|}
\hline \multirow[t]{2}{*}{ Patients } & \multirow[t]{2}{*}{$\begin{array}{l}\text { Agel } \\
\text { sex }\end{array}$} & \multirow[t]{2}{*}{ PEX } & \multirow[t]{2}{*}{$H T$} & \multirow[t]{2}{*}{$\begin{array}{c}\text { RPSV } \\
(>150 \mathrm{~cm} / \mathrm{s})\end{array}$} & \multirow[t]{2}{*}{$\begin{array}{l}R A R \\
(>3)\end{array}$} & \multicolumn{2}{|c|}{$\begin{array}{l}\text { Aneurysm of } \\
\text { abdominal aorta }\end{array}$} & \multicolumn{2}{|c|}{$\begin{array}{l}\text { Renal artery } \\
\text { stenosis }\end{array}$} \\
\hline & & & & & & Localization & $\begin{array}{c}\text { Diameter } \\
(\mathrm{mm})\end{array}$ & Localization & $\begin{array}{l}\text { Degree of } \\
\text { stenosis (\%) }\end{array}$ \\
\hline 1 & $56 / F$ & + & + & + & + & & & Right, orifice & 50 \\
\hline 2 & $69 / \mathrm{M}$ & + & + & + & + & & & Right, orifice & 50 \\
\hline 3 & $71 / \mathrm{F}$ & + & + & + & + & & & Left, orifice & 60 \\
\hline 4 & $87 / \mathrm{M}$ & + & + & + & + & Infrarenal & 40 & $\begin{array}{l}\text { Right, orifice and } \\
\text { hilar }\end{array}$ & 60,70 \\
\hline 5 & $68 / F$ & + & + & + & - & & & Right, hilar ${ }^{a}$ & 60 \\
\hline 6 & $62 / \mathrm{M}$ & + & + & + & + & Infrarenal & 40 & Left, orifice $^{b}$ & 70 \\
\hline 7 & $78 / \mathrm{M}$ & + & - & - & - & Infrarenal & 60 & & \\
\hline 8 & $88 / \mathrm{M}$ & + & - & + & + & & & Left, orifice & 60 \\
\hline 9 & $63 / \mathrm{M}$ & + & - & + & - & & & Right, hilar ${ }^{c}$ & 50 \\
\hline 10 & $81 / \mathrm{M}$ & + & - & + & + & & & Left, orifice & 50 \\
\hline 11 & $79 / \mathrm{M}$ & + & - & - & - & Infrarenal & 33 & & \\
\hline 12 & $67 / \mathrm{M}$ & + & - & - & + & & & $\mathrm{N}$ & \\
\hline 13 & $73 / \mathrm{M}$ & + & - & + & - & & & $\mathrm{N}$ & \\
\hline 14 & $81 / \mathrm{F}$ & - & + & - & + & & & Left, orifice & 60 \\
\hline 15 & $51 / \mathrm{M}$ & - & + & + & - & & & $\mathrm{N}$ & \\
\hline 16 & $70 / \mathrm{M}$ & - & + & + & - & & & $\mathrm{N}$ & \\
\hline
\end{tabular}

Abbreviations: CT, computed tomography; F, female; HT, hypertension; M, male; PEX, pseudoexfoliation syndrome; RAR, renoaortic ratio; RPSV, renal artery peak systolic velocity.

${ }^{\text {a }}$ Duplicated renal artery, inferior pole.

${ }^{\mathrm{b}}$ Double renal artery, superior.

${ }^{c}$ Duplicated renal artery, inferior pole.

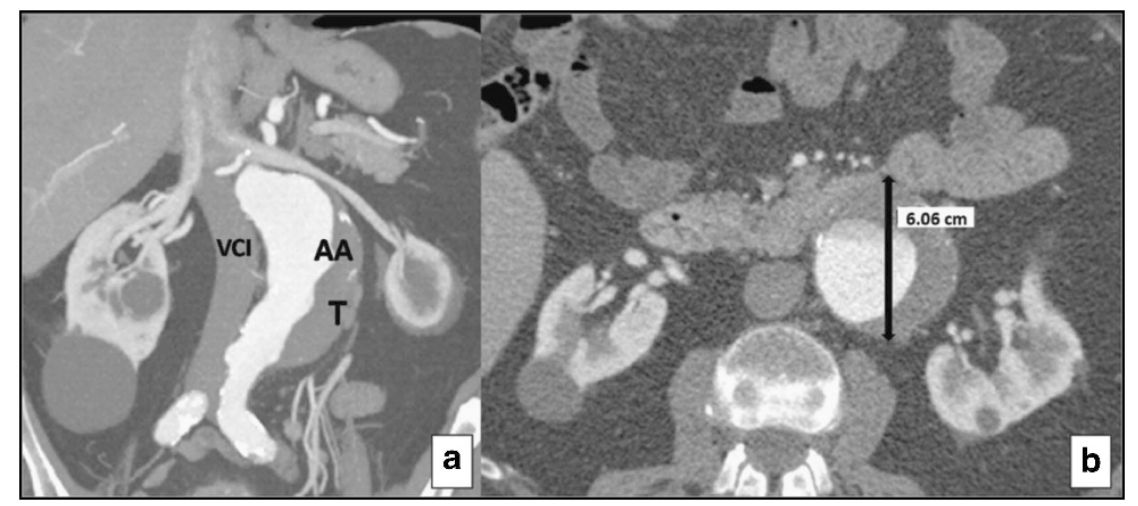

Figure 2 A 78-year-old male patient with infrarenal abdominal aorta aneurysm in PEX group. (a) Coronal CT reformat image shows a thrombosed abdominal aortic aneurysm. AA, abdominal aorta; T, thrombus; VCI, vena cava inferior. (b) Axial CT reformat image shows a thrombosed abdominal aortic aneurysm $6 \mathrm{~cm}$ in diameter.

that the overproduction and abnormal metabolism of the glycosaminoglycans, together with abnormally increased synthesis and the deposition of the elastotic fibrillar material in the tissues, have an important role in the pathophysiology of PEX. ${ }^{20-23}$ The prevalence of this syndrome increases with age. The relationship between PEX and vascular diseases has been investigated in many studies. $4,6,7,8,24-29$ However, there are also studies reporting that PEX is not associated with arterial hypertension, ischemic heart disease, and cerebrovascular diseases. ${ }^{27,30-34}$
Arterial hypertension is an important risk factor for the progression of cardiovascular diseases, stroke, and chronic renal diseases. Essential hypertension is the most common form of hypertension. Renovascular hypertension is present in $<5 \%$ of all causes of hypertension, however, it keeps an important place among the reasons of secondary hypertension. ${ }^{17,35}$ Recognition of the patients with RAS-induced hypertension has significant clinical implications due to correction of RAS with angioplasty, and stenting can improve blood pressure control in as many as $64 \%$ of 
patients with hypertension resistant to medical treatment. ${ }^{36}$ In addition, improvement of renal function occurs in $79 \%$ of patients in whom RAS is associated with deteriorating renal function. ${ }^{37}$ RAS is accounted for up to $15 \%$ of the patients with chronic renal failure. ${ }^{38}$ Thus, it is important to identify an accurate, noninvasive screening investigation for the detection of RAS. Although catheter angiography is accepted as the golden standard in the assessment of renal vessels, CT and magnetic resonance (MR) angiography are accepted as the alternative methods. ${ }^{17}$ The morbidity associated with catheter angiography from bleeding, anaphylaxis, and contrastinduced nephropathy has largely limited its use. Despite CT angiography has a potential risk of radiation toxicity and is contraindicated in patients with renal insufficiency, it provides excellent quality images with higher resolution than MR angiography. ${ }^{38}$ Doppler US is a cheap and noninvasive technique. There is no risk of ionizing radiation exposure. It provides valuable information about anatomy and physiology of the vascular structure. However, experience of the specialist and the cooperation of the patient are extremely important.

The most important Doppler US finding of RAS is the increase in flow velocity and turbulence at the point of stenosis. PSV over $180-200 \mathrm{~cm} / \mathrm{s}$ should be taken as highly suspicious. ${ }^{14,17}$ However, threshold values for studies in which PSV was evaluated ranged from 100 to $200 \mathrm{~cm} / \mathrm{s} .{ }^{16,17}$ RAR, which is the ratio of the RPSV to the APSV, is used for the exclusion and correction of the individual velocity variations. For cases with RAR values $>3-3.5$, one should be suspicious for the presence of a hemodynamically significant stenosis, and further investigations should be recommended. ${ }^{14,38,39}$ RAPSV and RAR are direct Doppler parameters of RAS. The threshold value of RAS that gives rise to hypertension or ischemic damage varies from patient to patient, nevertheless, a $50-60 \%$ stenosis in the arterial diameter is accepted to be hemodynamically significant. ${ }^{14,38}$ The threshold values that we used in our study for RAPSV and RAR were $150 \mathrm{~cm} / \mathrm{s}$ and 3, respectively. Among 14 patients who met the RAS criteria with Doppler US and who underwent CT angiography, 9 and 1 patients from the PEX and control groups, respectively, were found to have RAS of $50 \%$ or more. This difference between the groups was statistically significant. Six patients with RAS in the PEX group and one patient with RAS in the control group had hypertension.

Determination of RAS in $35.3 \%$ of the hypertensive patients in the PEX group may be considered an important relationship between PEX and hypertension associated with renovascular disease.

The most commonly used indirect Doppler US parameters in the establishment of RAS are the systolic acceleration ratio, the acceleration time, and the resistivity index ratio between the kidneys. ${ }^{16}$ However, as the results of these parameters are not as sensitive as the direct parameters, ${ }^{16}$ we did not prefer to use them in our study.

It has been demonstrated in the angiographic studies that $12-22 \%$ of the kidneys are perfused by more than one renal artery. Stenoses in the duplicate renal arteries may give rise to hypertension and renal insufficiency, however, small polar accessory arterial stenoses rarely lead to hypertension and do not give way to significant renal insufficiency. ${ }^{40}$ On the other hand, Hansen et al ${ }^{41}$ have also demonstrated that accessory renal arterial stenoses may be a reason for renovascular hypertension. In our study, the right renal arteries of two patients in the PEX group were duplicated at the distal ends. One of the patients with the RAS value of $60 \%$ was hypertensive, whereas the other with the RAS value of $50 \%$ was not. Another hypertensive patient in the PEX group had a left renal arterial duplication in which a $70 \%$ stenosis at the orifice of the superior renal artery was present.

As it is well known, $90-95 \%$ of stenotic or occlusive renal artery lesions are due to atherosclerosis or fibromuscular dysplasia. Although $75 \%$ of the atherosclerotic main renal artery stenoses occur either at the origin of the aorta (the orifice) or within the first centimeter of the vessel, $20 \%$ occurs at the trunk. Fibromuscular dysplasia occurs mainly at the middle and distal renal arteries in $60-70 \%$ of the cases and frequently reaches to more distal parts like the main branches of the renal arteries. ${ }^{15}$ RAS was at the orifice of the renal arteries in our eight patients, one of whom was in the control group. Because of this localization of RAS, especially in patient with PEX, we can consider a relationship between PEX and atherosclerosis. On the other hand in our selected patient group, because of absence of other factors that have a role in the development of atherosclerosis such as DM, chronic renal failure, heart disease, a history of smoking, and/or alcohol consumption, PEX can be considered to be an independent factor that may affect the renal vascular structures.

Although some articles report that there is a positive association between PEX and abdominal aorta aneurysm, others indicate to the contrary. ${ }^{7,11,29,30}$ In our study, four patients with PEX had abdominal aorta aneurysm, and in one of them, the abdominal aorta diameter was $6 \mathrm{~cm}$ (Figure 2). Nevertheless, none of the patients in the control group had an abdominal aorta aneurysm.

According to the best of our knowledge, the relationship between RAS and PEX has not previously been investigated. This study has demonstrated that there is a strong association between PEX and RAS. PEX 
can give rise to renovascular disease and abdominal aorta aneurysm, and may be accepted as an important marker of the renovascular pathology in hypertensive patients who are frequently accepted to have an idiopathic basis. Based on the results of current study, we definitely recommend investigation for RAS in all subjects with PEX, particularly those with hypertension.

\section{Summary}

What was known before

- Pseudoexfoliation syndrome is associated with cardiovascular, cerebrovascular, and peripheral vascular diseases.

What this study adds

- There is a significant association between pseudoexfoliation syndrome and renal artery stenosis.

\section{Conflict of interest}

The authors declare no conflict of interest

\section{Acknowledgements}

We are grateful to Pelin Toprakal Gok for assistance in this project. There were no sources of public and private financial support.

\section{References}

1 Schlötzer-Schrehart UM, Koca MR, Naumann GO, Volkholz H. Pseudoexfoliation syndrome. Ocular manifestation of a systemic disorder? Arch Ophthalmol 1992; 110: 1752-1756

2 Ritch R, Schlötzer-Schrehardt U. Exfoliation syndrome. Surv Ophthalmol 2001; 45: 265-315.

3 Ritch R. Exfoliation syndrome-the most common identifiable cause of open-angle glaucoma. J Glaucoma 1994; 3: 176-177.

4 Praveen MR, Shah SK, Vasavada AR, Diwan RP, Shah SM, Zumkhawala BR et al. Pseudoexfoliation as a risk factor for peripheral vascular disease: a case-control study. Eye (Lond) 2011; 25: 174-179.

5 Repo LP, Terasvirta ME, Koivisto KJ. Generalized transluminance of the iris and the frequency of the pseudoexfoliation syndrome in the eyes of transient ischemic attack patients. Ophthalmology 1993; 100: 352-355.

6 Mitchell P, Wang JJ, Smith W. Association of pseudoexfoliation syndrome with increased vascular risk. Am I Ophthalmol 1997; 124: 685-687.

7 Schumacher S, Schlötzer-Schrehardt U, Martus P, Lang W, Naumann GO. Pseudoexfoliation syndrome and aneurysms of the abdominal aorta. Lancet 2001; 357: 359-360.

8 Bojic L, Ermacora R, Polic S, Ivanisevic M, Mandic Z, Rogosic V et al. Pseudoexfoliation syndrome and asymptomatic myocardial dysfunction. Graefes Arch Clin Exp Ophthalmol 2005; 243: 446-449.
9 Linner E, Popovic V, Gottfries C, Jonsson M, Sjögren M, Wallin A. The exfoliation syndrome in cognitive impairment of cerebrovascular or Alzheimer's type. Acta Ophthalmol Scand 2001; 79: 283-285.

10 Cahill M, Early A, Stack S, Blayney AW, Eustace P. Pseudoexfoliation and sensorineural hearing loss. Eye 2002; 16: 261-266.

11 Djordjevic-Jocic J, Jovanovic P, Bozic M, Tasic A, Rancic Z. Prevalence and early detection of abdominal aortic aneurysm in pseudoexfoliation syndrome and pseudoexfoliation glaucoma. Curr Eye Res 2012; 37: 617-623.

12 Dawson DL. Noninvasive assessment of renal artery stenosis. Semin Vasc Surg 1996; 9: 172-181.

13 Aristizabal D, Frohlich ED. Hypertension due to renal arterial disease. Heart Dis Stroke 1992; 1: 227-234.

14 House MK, Dowling RJ, King P, Gibson RN. Using Doppler sonography to reveal renal artery stenosis: an evaluation of optimal imaging parameters. AJR Am J Roentgenol 1999; 173: 761-765.

15 Hillman BJ. Imaging advances in the diagnosis of renovascular hypertension. AJR Am J Roentgenol 1989; 153: 5-14.

16 Chain S, Luciardi H, Feldman G, Berman S, Herrera RN, Ochoa $\mathrm{J}$ et al. Diagnostic role of new Doppler index in assessment of renal artery stenosis. Cardiovasc Ultrasound 2006; 25: 4 .

17 Williams GJ, Macaskill P, Chan SF, Karplus TE, Yung W, Hodson EM et al. Comparative accuracy of renal duplex sonographic parameters in the diagnosis of renal artery stenosis: paired and unpaired analysis. AJR Am J Roentgenol 2007; 188: 798-811

18 Streeten BW, Li ZY, Wallace RN, Eagle Jr, RC, Keshgegian AA. Pseudoexfoliative fibrillopathy in visceral organs of a patient with pseudoexfoliation syndrome. Arch Ophthalmol 1992; 110: 1757-1762.

19 Naumann GO, Schlötzer-Schrehart U, Küchle M. Pseudoexfoliation syndrome for the comprehensive ophthalmologist: intraocular and systemic manifestation. Ophthalmology 1998; 105: 951-968.

20 Schlötzer-Schrehardt U, Dörfler S, Naumann GO. Immunohistochemical localization of basement membrane components in pseudoexfoliation material of the lens capsule. Curr Eye Res 1992; 11: 343-355.

21 Davanger M. On the interfibrillar matrix of the pseudoexfoliation material. Acta Ophthalmol 1978; 56: 233-240.

22 Seland JH. The ultrastructural changes in the exfoliation syndrome. Acta Ophthalmol 1988; 184: 28-34.

23 Bergmanson JP, Jones WL, Chu LW. Ultrastructural observations on (pseudo-) exfoliation of the capsule: a re-examination of involvement of the lens epithelium. Br J Ophthalmol 1984; 68: 118-123.

24 Citirik M, Acaroglu G, Batman C, Yildiran L, Zilelioglu O. A possible link between the pseudoexfoliation syndrome and coronary artery disease. Eye (Lond) 2007; 21: 11-15.

25 Repo LP, Suhonen MT, Terasvirta ME, Koivisto KJ. Color Doppler imaging of the ophthalmic artery blood flow spectra of patients who have had transient ischemic attack. Correlations with generalized iris transluminance and pseudoexfoliation syndrome. Ophthalmology 1995; 102: 1119-1205.

26 Akarsu C, Unal B. Cerebral haemodynamics in patients with pseudoexfoliation glaucoma. Eye (Lond) 2005; 19: 1297-1300. 
27 Ritland JS, Egge K, Lydersen S, Juul R, Semb SO. Exfoliative glaucoma and primary open-angle glaucoma: associations with death causes and comorbidity. Acta Ophthalmom Scand 2004; 82: 401-404.

28 Yüksel N, Anik Y, Altintaş O, Onur I, Cağlar Y, Demirci A. Magnetic resonance imaging of the brain in patients with pseudoexfoliation syndrome and glaucoma. Ophthalmologica 2006; 220: 125-130.

29 Hietanen J, Soisalon-Soininen S, Kivelä T, Tarkkanen A. Evaluation of the clinical association between exfoliation syndrome and abdominal aortic aneurysm. Acta Ophthalmol Scand 2002; 80: 617-619.

30 Tarkkanen A, Reunanen A, Kivelä T. Frequency of systemic vascular diseases in patients with primary open-angle glaucoma and exfoliation glaucoma. Acta Ophthalmol 2008; 86: 598-602.

31 Allingham RR, Loftsdottir M, Gottfredsdottir MS, Thorgeirsson E, Jonasson F, Sverisson T et al. Pseudoexfoliation syndrome in Icelandic families. Br J Ophthalmol 2001; 85: 702-707.

32 Shingleton BJ, Heltzer J, O'Donoghue MW. Outcomes of phacoemulsification in patients with and without pseudoexfoliation syndrome. J Cataract Refract Surg 2003; 29: $1080-1086$

33 Ringvold A, Blika S, Sandvik L. Pseudo-exfoliation and mortality. Acta Ophthalmol Scand 1997; 75: 255-256.
34 Shrum KR, Hattenhauer MG, Hodge D. Cardiovascular and cerebrovascular mortality associated with ocular pseudoexfoliation. Am J Ophthalmol 2000; 129: 83-86.

35 Sidhu R, Lockhart ME. Imaging of renovascular disease. Semin Ultrasound CT MR 2009; 30: 271-288.

36 Jensen G, Zachrisson BF, Delin K, Volkmann R, Aurell M. Treatment of renovascular hypertension: one year results of renal angioplasty. Kidney Int 1995; 48: 1936-1945.

37 Bonelli FS, McKusick MA, Textor SC, Kos PB, Stanson AW, Johnson CM et al. Renal artery angioplasty: technical results and clinical outcome in 320 patients. Mayo Clin Proc 1995; 70: 1041-1052.

38 Claudon M, Plouin PF, Baxter GM, Rohban T, Devos DM. Renal arteries in patients at risk of renal arterial stenosis: multicenter evaluation of the echo-enhancer SH U 508A at color and spectral Doppler US. Levovist Renal Artery Stenosis Study Group. Radiology 2000; 214: 739-746.

39 Miralles M, Cairols M, Cotillas J, Giménez A, Santiso A. Value of Doppler parameters in the diagnosis of renal artery stenosis. J Vasc Surg 1996; 23: 428-435.

40 Zwiebel WJ. Introduction to Vascular Ultrasonography, 3rd edn. WB Saunders Co: Philadelphia, PA, 1992.

41 Hansen KJ, Tribble RW, Reavis SW, Canzanello VJ, Craven TE, Plonk Jr, GW et al. Renal duplex sonography: evaluation of clinical utility. J Vasc Surg 1990; 12: 227-236. 